\title{
Extended Colectomy Followed by Cecorectal Anastomosis as a Surgical Treatment Modality in Synchronous Colorectal Cancer
}

\author{
Sergey K. Efetov ${ }^{a} \quad$ Abina A. Zubayraeva ${ }^{a} \quad$ Valery M. Nekoval $^{a}$ \\ Aleksandra S. Tyan ${ }^{b}$ Inna A. Tulina ${ }^{a}$ Petr V. Tsarkov ${ }^{a}$ \\ ${ }^{a}$ Coloproctology and Minimally Invasive Surgery Clinic, I.M. Sechenov First Moscow State \\ Medical University (Sechenov University), Moscow, Russia; ${ }^{b}$ Department of Radiology and \\ Radiotherapy, I.M. Sechenov First Moscow State Medical University (Sechenov University), \\ Moscow, Russia
}

\author{
Keywords \\ Synchronous cancer - Extended colectomy · Cecorectal anastomosis · Colorectal cancer
}

\begin{abstract}
Subtotal and extended left colectomies with ileocecal junction preservation represent preferable alternatives in cases of massive involvement of the colon in the pathological process. However, these approaches might be challenging in terms of reconstructive steps. Antiperistaltic cecorectal anastomosis is one of the possible techniques. Still, this type of pouch formation is described mostly in slow-transit constipation surgical management. We report on a patient with synchronous colorectal cancer who underwent extended left colectomy. In the case of compromised vessel anatomy, it was decided to perform antiperistaltic cecorectal anastomosis. We present all clinical and intraoperative patient's data, determining the surgical tactics, and short-term postoperative results. An antiperistaltic cecorectal anastomosis can be considered in nonstandard clinical cases and variable anatomy of the patient.
\end{abstract}

(C) 2020 The Author(s)

Published by S. Karger AG, Basel

\section{Introduction}

Extended hemicolectomies, as well as subtotal colectomies, are the possible options for salvage surgical treatment in specific clinical scenarios. These procedures were performed in patients with diverticulitis, Crohn's disease, transverse carcinoma, synchronous cancer, peritoneal carcinomatosis, previous sigmoidectomy, polyposis, and intractable constipation [1-3]. These approaches are considered to be preferable in these cases, as total colectomy can result in decreased reabsorption of water and electrolytes, ending up with diarrhea [4]. 
The reconstructive procedures after extended left colectomy require the formation of a tension-free colorectal anastomosis, saving the terminal ileum, ileocecal valve, and cecum when possible. The preservation of the colonic segment is crucial regarding the decrease of bowel motions and lower risk of postoperative small bowel obstruction and anastomotic leakage [5].

Mostly, the reconstruction is feasible with a right colonic transposition or a complete intestinal derotation, known as Deloyers procedure $[5,6]$. The colonic rotation might be complicated by the variability of vessel anatomy and difficulty in supplying artery preservation [7].

Cecorectal anastomosis is also a possible option. Its efficacy is described in several reports $[1,2]]$. Still, there is a limited number of papers describing the cecorectal anastomosis as a reconstructive procedure after extensive colorectal surgery for different pathological conditions.

In this paper, we provide an overview of the indications for subtotal and extended colectomies, cecorectal anastomosis as a reconstructive stage of the procedure, and discuss the technical aspects and functional outcomes, described previously. Moreover, we introduce our experience in cecorectal anastomosis formation in a patient with synchronous colorectal cancer.

\section{Case Presentation}

\section{Preoperative Assessment}

A 73-year-old patient was admitted to the Department of Coloproctology with descending colon cancer diagnosed previously and initial complaints on paraumbilical pain and weakness.

The colonoscopy showed multiple epithelial neoplastic lesions in the transverse and sigmoid colon, laterally spreading tumor in the hepatic flexure, and lesions in the descending colon and rectum. The histological examination of the biopsies proved the presence of tubulovillous adenomas in the proximal parts of the transverse and sigmoid colon and moderately differentiated adenocarcinoma of the transverse and descending colon and rectum.

MRI showed the tumor in the middle rectum $73 \mathrm{~mm}$ above the external anal sphincter verge, infiltrating all bowel layers and invading into the mesorectum, as well as transverse and descending colon tumors with subserosa invasion.

On contrast-enhanced CT, a lesion was detected in the middle and low rectum as a concentric wall thickening $15 \mathrm{~mm}$ in length and adjacent mesorectal fat stranding. Also, there were contrast-enhanced tumors in the transverse colon close to the hepatic flexure and in the proximal part of the descending colon. No distant metastases were found. Despite the synchronous tumor spread, Lynch syndrome and constitutional mismatch repair deficiency were disproved (no loss of MSH2, MSH6, PMS2, and MLH1 was identified).

The final diagnosis was laterally spreading tumor of the hepatic flexure, multiple primary synchronous stage III adenocarcinomas of the descending colon and rectum (cT3N1M0 and cT3N2M0, respectively).

\section{The Surgical Procedure}

The tumors of the proximal thirds of the transverse and descending colon were confirmed during intraoperative revision. Malignant transformation was suspected because of the large diameter of the palpable laterally spreading tumor $(3 \mathrm{~cm})$ in the hepatic flexure and the presence of villous components identified during pathological assessment of the biopsies. In the absence of the histological evaluation of the entire polyps and tumors, it was decided to 

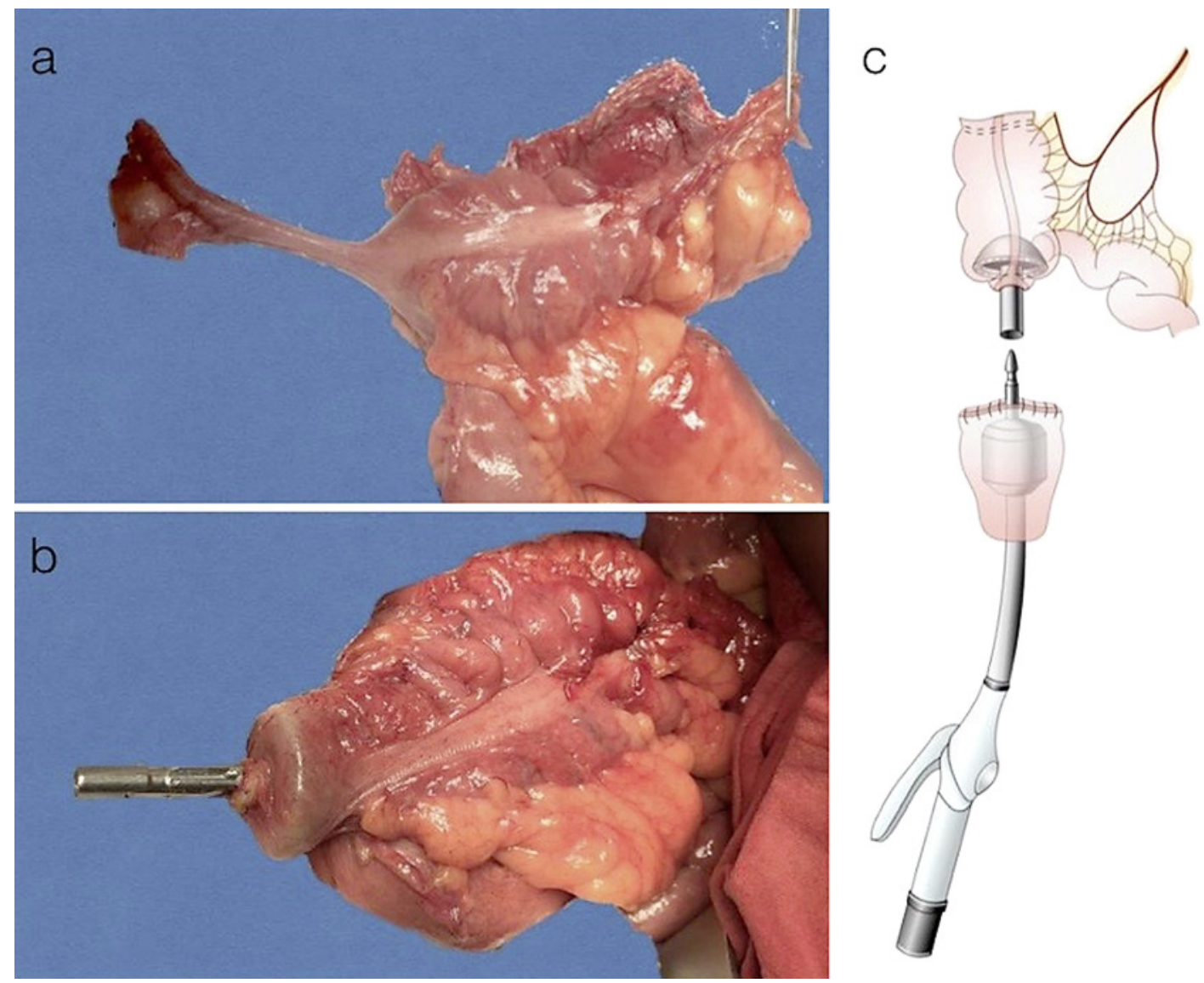

Fig. 1. Cecorectal anastomosis with transanal assistance. a Ileocecal segment mobilized. b Anvil shaft exerted through the appendiceal orifice. c Scheme of the anastomosis.

perform the extended left hemicolectomy and low anterior resection of the rectum with D3 lymph node dissection (D3 LND) to follow the oncological principles of colon resection. We adopted a 10-cm margin from the marked tumor in the hepatic flexure according to Japanese guidelines [8].

The extended left colectomy combined with low anterior resection of the rectum and extended D3 LND was performed. The high ligation of the inferior mesenteric artery and medial colic artery was achieved, the segment of the colon and rectum removed.

Since the location of the ileocolic artery was more cranial than usual, supplying the stump of the ascending colon right at the line of resection approximately $7 \mathrm{~cm}$ above the cecum, which limited both transposition and derotation, it was decided to perform an antiperistaltic cecorectal anastomosis. Firstly, the cecum was mobilized (Fig. 1a) and lowered into the pelvis. The appendectomy was performed. The anvil of Ethicon $29 \mathrm{~mm}$ Curved Intraluminal Stapler (CDH29A) was inserted into the colonic stump with the anvil shaft exerted through the appendiceal orifice (Fig. 1b) and fixated with a purse-string suture. The stump of the ascending colon was closed with Ethicon Linear Cutter Stapler (NTLC75). The intraluminar circular stapler was introduced via the anal canal (Fig. 1c). The end-to-end cecorectal anastomosis was performed between the fundus of the cecum and the remaining part of the rectum $5 \mathrm{~cm}$ above the anal verge, i.e., $2 \mathrm{~cm}$ below the inferior border of the tumor) with a defunctioning loop ileostomy by Turnbull. The total blood loss was $150 \mathrm{~mL}$. 

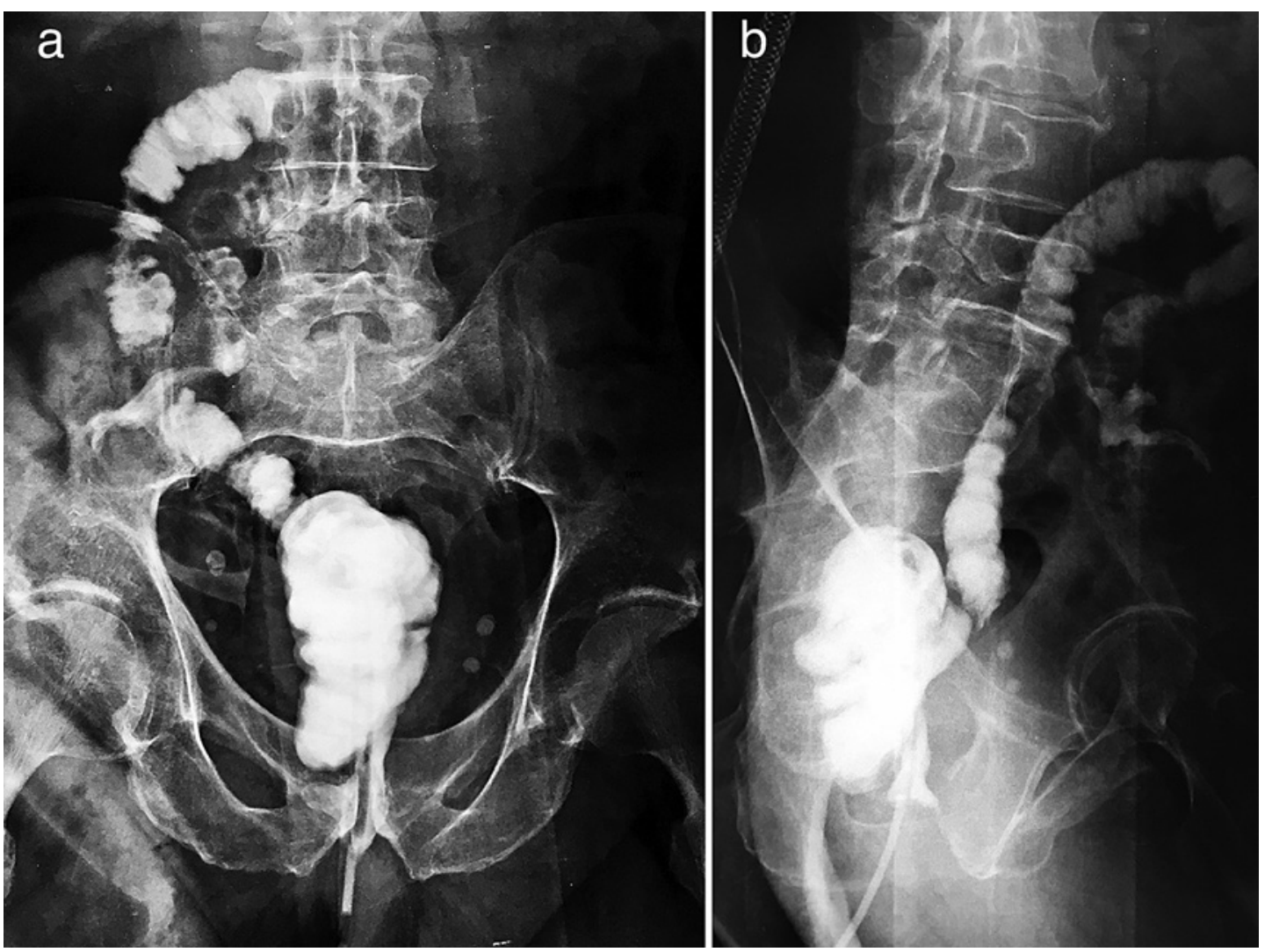

Fig. 2. Water-soluble agent enema shows the cecorectal pouch. a Frontal plane. b Sagittal plane.

The postoperative pathological staging proved multiple primary synchronous colorectal cancer of the descending colon pT2N1b and rectum pT3N1b, other lesions were identified as benign.

\section{Postoperative Assessment}

The functionality of the pouch was assessed by evacuation proctography 1 month postoperatively with radiocontrast agent Omnipaque ${ }^{\circledR}$. The water-soluble agent enema showed colon pouch configuration and the absence of anastomotic side leakage (Fig. 2a, b).

The postoperative length of stay was 11 days. The temporary stoma was closed 8 weeks after the initial surgery. The patient said that during the first week after stoma closure, the stool was normally shaped and consistent (types 3 and 4 according to Bristol stool chart). The patient reported on up to 4 bowel movements per day reaching a regular pattern and uncontrollable defecation 1 month after stoma closure. The quality of life was evaluated by the Gastrointestinal Quality of Life Index (GIQLI), the defecation was assessed via Wexner-Vaizey fecal incontinence score [9] and Diarrhea Assessment Score (DAS) 3 months postoperatively (Table 1).

\section{Discussion}

Usually, when the colon and rectum are severely affected by a pathological process, coloproctectomy is performed. Total colectomy with ileorectal anastomosis was reported to be followed by diarrhea, persistence of constipation, and increased number of evacuations [4]. 


\section{Case Reports in Oncology}

Table 1. Questionnaires for postoperative assessment

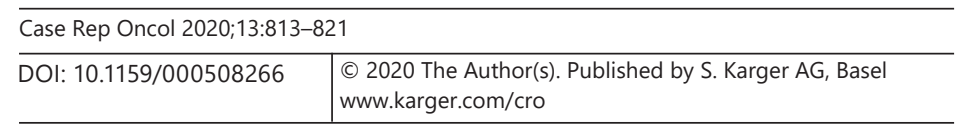

\begin{tabular}{lcl}
\hline Questionnaire & Score & Distribution, \% \\
\hline GIQLI (0-144), including & 80 & 69.4 \\
(1) Symptoms (76) & 56 & 56 \\
(2) Physical status (28) & 15 & 13 \\
(3) Emotional status (20) & 13 & 16 \\
(4) Social status (20) & 16 & 15 \\
Wexner-Vaizey score (0-24) & 14 & 58.3 \\
DAS (0-12) & 6 & 50 \\
\hline
\end{tabular}

GIQLI: Gastrointestinal Quality of Life Index; DAS: Diarrhea Assessment Score.

Mostly, subtotal and extended colectomies are described for slow-transit constipation surgical management. They may become procedures of choice in specific clinical cases in patients with slow-transit constipation, ulcerative colitis, polyposis, transverse carcinoma, and synchronous cancer when part of the ascending colon, cecum and terminal ileum can be saved. Because of poor functional results after total colectomies, Sarli et al. [10] proposed the subtotal colectomy with antiperistaltic cecoproctostomy in the treatment of chronic constipation.

\section{Specificities of Subtotal and Extended Left Colectomy in the Surgical Treatment of}

Slow-Transit Constipation and Synchronous Colorectal Carcinomas

Some authors suggest extended left colectomy or subtotal colectomy with cecum preservation, claiming that terminal ileum, ileocecal valve, and cecum are crucial in terms of water, bile, and electrolytes absorption, playing a significant role in decreased risk of diarrhea and anastomotic failure [5]. Marchesi et al. [2] showed that the preservation of the ileocecal valve and cecal pouch decreased the risk of postoperative incontinence. Moreover, they compared the non-slow-transit constipation group, which included patients with carcinomas, polyposis, ulcerative colitis, Crohn's disease, extended diverticular disease, and radiation injuries, to those with slow-transit constipation, treated both with laparoscopic and open surgery. The functional outcomes, such as the Wexner Score and Gastrointestinal Quality of Life Index, were mostly the same in both groups, with no statistically significant difference. The latter surveillance confirms the applicability of cecorectal anastomosis in patients with colonic lesions and inflammatory diseases.

Also, some cases describe the mandatory role of these procedures in treating polyposis, synchronous cancer, and carcinomatosis.

Heneghan et al. [11] compared subtotal or segmental colectomies (therapeutic) to total ones (prophylactic) in the treatment of hereditary nonpolyposis colorectal cancer (HNPCC). It was reported that there was a higher incidence of metachronous adenomas and cancer after therapeutic colectomies ( $p=0.057$ and $<0.005$, respectively). Still, it did not affect longterm survival ( $90.7 \%$ after segmental and $89.8 \%$ after total colectomy, $p=0.085$ ), so the prophylactic colectomy is contentious in the treatment of HNPCC. However, most of the observations are single-center studies limited by the retrospective nature and small patient cohort size.

The first cases of subtotal colectomy with cecorectal anastomosis in multiple adenomas treatment were reported in 1962 by Rosi and Cahill [3]. Sarli et al. [1] demonstrated that patients with inflammatory diseases and colon tumors could benefit from subtotal colectomies with ileocecal junction preservation. By the 4.5-year mean follow-up period, these patients had satisfactory results regarding frequency of bowel movements with 1.7 per day 
and normal stool consistency. So, subtotal colectomy with cecoproctostomy may be even more favorable for inflammatory and neoplastic disorders treatment, rather than in patients with slow-transit constipation. This procedure may also be considered in selective clinical cases with specific anatomical features, confining the surgical tactics.

Jones et al. [7] performed anastomotic cecorectal reconstruction with middle colic artery preservation in patients with colovesical fistula en bloc resection. The compromised vessel anatomy and the aim to save the remaining mid-transverse colon were the main reasons to perform antiperistaltic cecorectal anastomosis with long pouch. Wei et al. [12] introduced their experience on cecorectal anastomosis with short and long colonic reservoir and their comparative analysis. They observed that abdominal pain and bloating scale were more significant in patients with pouches of $10-15 \mathrm{~cm}$ in length. On the barium enema, it was found that the bind loop-pouch was the cause for increased intensity of abdominal pain and bloating. The shortening of the pouch caused the release of patients' complaints. It was concluded that shorter pouches result in better postoperative outcomes.

\section{Types of Reconstructive Techniques following Subtotal and Extended Left Colectomies}

The possible approaches following the extensive surgery of the left colon include both iso- or antiperistaltic anastomosis formation. The first one requires the lowering of the cecum and colon position changing to make the anastomosis with the rectum or anal canal. For that purpose, retroileal transmesenteric anastomosis is widely performed. First described by Andre Toupet in 1961, it was actively performed by Rombeau et al. [13] in 11 patients with cancer and diverticulitis after extended left hemicolectomies, describing this approach as more preferable in comparison to ileorectal anastomosis, especially in elderly people with weak anal sphincter. The patients did not develop any feces incontinence, regardless of poor preoperative sphincter function. The technique itself is performed to avoid tension of the mobilized colon, which is pulled through a bluntly dissected mesenteric window. The window is created behind the most distal ileal loop in the avascular space $[13,14]$.

The counterclockwise rotation of the cecum and ascending colon, known as "Deloyers procedure," was introduced by Rosi and Cahill [3] in 1962 and Lucien Deloyers in 1964, as a possible reconstructive technique after extended left hemicolectomy and subtotal colectomy. The procedure consists of colonic mobilization, craniocaudal rotation, and positioning in the iliac fossa (Fig. 3b) [3, 6]. In the modification, presented earlier by Lillehei and Wangensteen [15], the rotation vector passes through the horizontal plane in $180^{\circ}$ after craniocaudal transposition of the cecum (Fig. 3a). The third approach, suggested by Ryan and Oakley [16], is an end-to-side anastomosis, which is performed after $90^{\circ}$ transposition both in the sagittal and horizontal planes (Fig. 3c). However, these techniques have certain limitations. Regarding the transmesenteric approach, the window formation may be challenging in obese patients and in those operated laparoscopically. Moreover, there is a risk of herniation and small bowel obstruction. The approaches, requiring bowel rotation or transposition, may be limited by compromised anatomical features of the bowel blood supply, as they require the twisting of the mesenteric root and the vascular pedicle within it.

In these cases, antiperistaltic cecorectal anastomosis may become a potential alternative. For the first time introduced by Sarli et al. [1] it is now commonly called "Sarli procedure" [9]. This type of anastomosis requires neither the transposition of the cecum and its lowering nor mesenteric root rotation (Fig. $3 \mathrm{~d}$ ). This procedure is widely performed in patients with slowtransit constipation. Marchesi et al. [2] conducted a comparative study in patients with slowtransit constipation and other pathologies as a control group, who seemed to benefit from this procedure. In our case, we performed the cecorectal anastomosis with ascending pouch formation by Ethicon $29 \mathrm{~mm}$ Curved Intraluminal Stapler (CDH29A) in the patient with synchronous colorectal cancer and multiple polyposis (Fig. 3e). The created pouch was $14 \mathrm{~cm}$ 


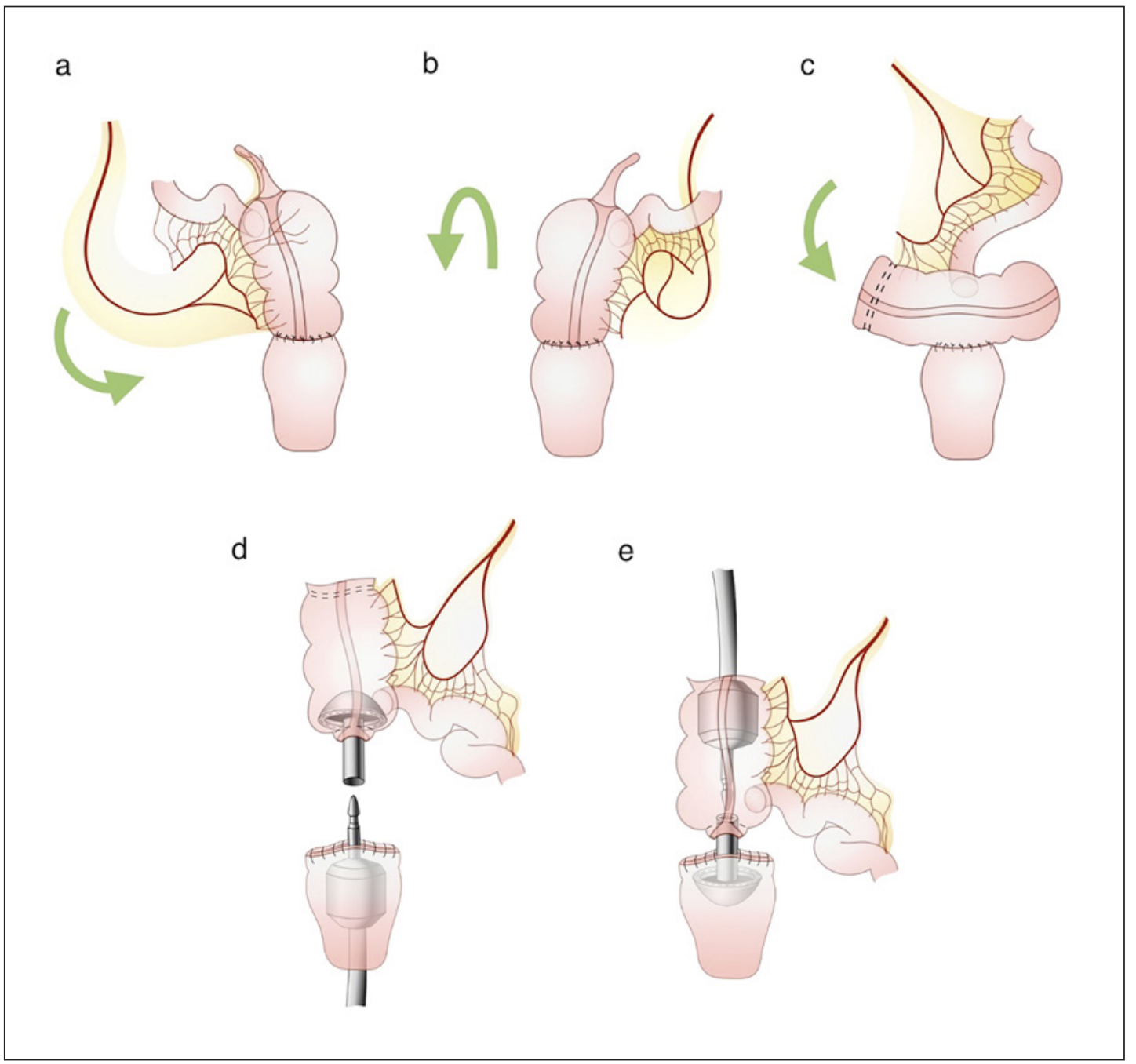

Fig. 3. Types of reconstructive surgery after subtotal colectomy with ileocecal junction preservation. a Isoperistaltic ascendo- or cecorectal anastomosis, proposed by Lillehei and Wangensteen [15]. b Deloyers procedure. c Ascendo- or cecorectal anastomosis side-to-end. d, e Cecorectal anastomosis in the case of the presented patient.

long with the ascending pouch around $7 \mathrm{~cm}$ in length. The anastomosis was performed via transanal introduction of the circular stapler, while the anvil was pushed through the appendiceal orifice. The main reason to perform this kind of restorative surgery was more cranial inflow of the supplying ileocolic artery (around $7 \mathrm{~cm}$ above the ileocecal junction), which limited the lowering of the ascending part of the preserved colon. The risk of vascular pedicle torsion is increased in Deloyers procedure or transmesenteric colorectal anastomosis.

The patient reported on a satisfying quality of life after surgery in comparison with the preoperative stage, as well as positive dynamics in stool frequency and consistency. According to questionnaires, there is still a slight severity of postoperative incontinence and diarrhea syndrome (Table 1), which can be explained by the short-term postoperative period, that is, 3 months after initial surgery and 1 month after stoma closure. A slightly lower percentage distribution in physical and emotional status, shown by GIQLI, and diarrhea syndrome occurrence can be caused by adjuvant therapy and the short length of the postoperative period. Marchesi et al. [2] demonstrated more promising functional outcomes and quality of life in 
non-slow-transit constipation patients starting 22 months after surgery. The GIQLI results vary from 63 to 138, Wexner Index ranges from 0 to 8 . Thus, long-term outcomes are needed to prove that this procedure is effective and safe in our clinical case.

\section{Conclusion}

Antiperistaltic cecorectal anastomosis is rarely performed in patients presenting with multiple adenomas and carcinomas; it is more routinely carried out in patients with colonic inertia. We suggest this modified approach in patients with synchronous colorectal cancer and polyposis after extended left colectomy with satisfactory perioperative functional outcomes (i.e., bowel movements and stool consistency). It is highly important to bear in mind and consider this procedure in selective patients with atypical anatomy or unusual clinical features, as it allows to save the ileocecal junction, which is important in terms of functional results.

\section{Statement of Ethics}

Written informed consent was obtained from the patient for this case report and all the accompanying pictures and data.

\section{Conflict of Interest Statement}

The authors declare that they have no conflicts of interest to disclose on the subject discussed in the paper.

\section{Funding Sources}

We do not have any funding sources to disclose.

\section{Author Contributions}

S.K. Efetov: first surgeon, conception of the study design, data collection and analysis, manuscript writing, revision, and submission. A.A. Zubayraeva: conception of the study, data collection and analysis, manuscript writing, revision, and submission. V.M. Nekoval: second surgeon, data collection and analysis, patient preoperative and postoperative assessment. A.S. Tyan: data collection and analysis, manuscript writing, revision, and submission, patient preoperative and postoperative assessment. I.A. Tulina: conception of the study, data collection and analysis, critical revision of the manuscript. P.V. Tsarkov: conception of the study design, data collection and analysis, manuscript writing and editing, the approval of the final version for the submission. 


\section{References}

1 Sarli L, Costi R, Iusco D, Roncoroni L. Long-term results of subtotal colectomy with antiperistaltic cecoproctostomy. Surg Today. 2003;33(11):823-7.

2 Marchesi F, Sarli L, Percalli L, Sansebastiano GE, Veronesi L, Di Mauro D, et al. Subtotal colectomy with antiperistaltic cecorectal anastomosis in the treatment of slow-transit constipation: Long-term impact on quality of life. World J Surg. 2007;31(8):1658-64.

3 Rosi PA, Cahill WJ. Subtotal colectomy with cecorectal anastomosis for multiple adenomas of the colon. Am J Surg. 1962;103(1):75-80.

4 Ripetti V, Caputo D, Greco S, Alloni R, Coppola R. Is total colectomy the right choice in intractable slow-transit constipation? Surgery. 2006;140(3):435-40.

5 Dumont F, Da Re C, Goéré D, Honoré C, Elias D. Options and outcome for reconstruction after extended left hemicolectomy. Colorectal Dis. 2013;15(6):747-54.

6 Manceau G, Karoui M, Breton S, Blanchet AS, Rousseau G, Savier E, et al. Right colon to rectal anastomosis (Deloyers procedure) as a salvage technique for low colorectal or coloanal anastomosis: postoperative and long-term outcomes. Dis Colon Rectum. 2012;55(3):363-8.

7 Jones DW, Dimitrova M, Nandakumar G. Colonic salvage with antiperistaltic cecorectal anastomosis. Dis Colon Rectum. 2015;58(2):270-4.

8 Watanabe T, Muro K, Ajioka Y, Hashiguchi Y, Ito Y, Saito Y, et al. Japanese Society for Cancer of the Colon and Rectum (JSCCR) guidelines 2016 for the treatment of colorectal cancer. Int J Clin Oncol. 2018;23(1):1-34.

9 Vaizey CJ, Carapeti E, Cahill JA, Kamm MA. Prospective comparison of faecal incontinence grading systems. Gut. 1999;44(1):77-80.

10 Sarli L, Costi R, Sarli D, Roncoroni L, Wexner SD. Pilot study of subtotal colectomy with antiperistaltic cecoproctostomy for the treatment of chronic slow-transit constipation. Dis Colon Rectum. 2001;44(10):1514-20.

11 Heneghan HM, Martin ST, Winter DC. Segmental vs extended colectomy in the management of hereditary nonpolyposis colorectal cancer: A systematic review and meta-analysis. Colorectal Dis. 2015;17(5):382-9.

12 Wei D, Cai J, Yang Y, Zhao T, Zhang H, Zhang C, et al. A prospective comparison of short term results and functional recovery after laparoscopic subtotal colectomy and antiperistaltic cecorectal anastomosis with short colonic reservoir vs. long colonic reservoir. BMC Gastroenterol. 2015;15(1): 30.

13 Rombeau JL, Collins JP, Turnbull RB. Left-sided Colectomy with Retroileal Colorectal Anastomosis. Arch Surg. 1978;113(8):1004-5.

14 Chen YC, Fingerhut A, Shen MY, Chen HC, Ke TW, Chang SJ, et al. Colorectal anastomosis after laparoscopic extended left colectomy: techniques and outcome. Colorectal Dis. 2020;0-2.

15 Lillehei RC, Wangensteen $\mathrm{OH}$. Bowel function after colectomy for cancer, polyps, and diverticulitis. J Am Med Assoc. 1955;159(3):163-70.

16 Ryan JA, Oakley WC. Cecoproctostomy. American J Surg. 1985;149(5):636-9. 